\begin{tabular}{ccc}
\hline & $\begin{array}{c}\text { International Journal of Health Services } \\
\text { Research and Policy } \\
\text { www.dergipark.org.trijhsrp }\end{array}$ \\
$\begin{array}{c}\text { INTERESATIONAL } \\
\text { ENGINEERING } \\
\text { SCIENCE AND } \\
\text { EDUCATION GROUP }\end{array}$ & e-ISSN: $2602-3482$ & IJHSRP \\
\hline
\end{tabular}

Research Article

\title{
INVESTIGATION OF SFLT-1 AND VEGF EXPRESSION IN NORMOTENSIVE AND PREECLAMPTIC PLACENTA. AN IMMUNOHISTOCHEMICAL STUDY
}

\section{Firat Sahin $^{1}$ (D) Murat Akkus $^{1}$ (D) Ugur Seker ${ }^{1}$ (D) $*$ Sevda Soker $^{1}$ (D) Ebru Gokalp Ozkorkmaz ${ }^{1}$ (D) Elif Agacayak $^{2}$ (D) Firat Asir ${ }^{1}$ (D)}

${ }^{1}$ Department of Histology and Embryology, Faculty of Medicine, Dicle University, Diyarbakir, Turkey.

${ }^{2}$ Department of Obstetrics and Gynecology, Faculty of Medicine, Dicle University, Diyarbakir, Turkey.

* Corresponding author: seker.ugur.tr@gmail.com

\begin{abstract}
The pathogenesis of preeclampsia is still not clear, but endothelial dysfunction is believed to be one of the most encountered problems during placenta development in preeclamptic patients. Both vascular endothelial growth factor (VEGF) and its antagonist, soluble Fms-Like tyrosine kinase1 (sFlt-1), have roles in vascular function. In this study, we have investigated the immunohistochemical expression of VEGF and sFlt-1 in term placenta of normotensive and preeclampsia patients. A total of twenty term placentas were obtained from pregnant women, of whom 10 were preeclampsia patients and 10 were normotensive patients. Placentas were dissected and tissue samples were subjected to routine tissue processing protocol, and then embedded in paraffin blocks. Serial sections were obtained from paraffin blocks and stained with $H \& E$ and PAS for routine histopathology. VEGF and sFlt-1 immunohistochemistry was performed on the sections. When compared to the control group, severe pathological changes were observed in preeclamptic placentas. An increase in the number of syncytial knots and intervillous bridges, hemorrhage in interstitium, dilatation, and congestion in villous capillaries, increase in fibrin accumulation in villous stroma, and increase in thickening of the basement membrane were very clear. VEGF expression was significantly higher in normotensive placenta compared to the preeclamptic placenta. On the other hand, sFlt-1 expression was significantly increased in preeclamptic placenta villous capillary endothelial cells. When the VEGF and sFlt-1 expression is considered, a higher expression of sFlt-1 at preeclampsia, but a decrease in VEGF expression might be related to endothelial dysfunction in preeclampsia. Overall, this study demonstrates that the imbalance between VEGF and sFlt-1 is one of the major reasons for endothelial dysfunction in the preeclamptic placenta.
\end{abstract}

Keywords: Preeclampsia, placenta, immunohistochemistry, VEGF, sFlt-1.

Received: July 9, 2020

Accepted: September 8, 2020

\section{Introduction}

Although some improvements have been made in its diagnosis and treatment, preeclampsia is still one of the most common causes of maternal and fetal morbidity and mortality in the whole world 
[1]. Each year, in developing countries, more than 60,000 mothers die due to preeclampsia-related complications [2]. Preeclampsia is a clinical syndrome that arises after the 20th week of gestation with clinical complications such as hypertension and proteinuria [3]. Fetal complications of preeclampsia can be listed as low birth weight, intrauterine growth restriction (IUGR), preterm birth, and fetal death. On the other hand, maternal complications can be listed as renal failure, HELLP (hemolysis, elevated liver enzyme levels, and low platelet levels) syndrome, liver failure, cerebral edema, and maternal death. Also, some evidence indicates that preeclampsia may be predisposed by various metabolic diseases $[4,5]$. Therefore, understanding the pathogenesis of preeclampsia will provide advantages in early diagnosis and treatment thereof. Until today, numerous studies have investigated the pathogenesis of preeclampsia, but the reasons underlying this syndrome haven't been clearly understood yet. The common belief is that there exist morphologic and functional defects in preeclamptic placentas. One of the most commonly revealed hypotheses is the incompleteness of placental angiogenesis and development which is believed to be resulting from endothelial dysfunction [6]. Appropriate placenta development is dependent on vasculogenesis processed with a sensitive balance between pro-and anti-angiogenic factors. Recent studies demonstrate that an imbalance of the volume of pro-and anti-angiogenic proteins in circulation leads to endothelial dysfunction in preeclampsia. Pro-angiogenic, vascular endothelial growth factor (VEGF) and anti-angiogenic, soluble fms-like tyrosine kinase-1 (sFlt-1), the two leading factors in angiogenesis, are believed to play a role in the mitosis of placental endothelial cells. According to this information, the imbalance between VEGF and sFlt-1 is believed to be one of the reasons for preeclamptic endothelial dysfunction. However, there is a conflict among previous studies in correlating the expression level of VEGF and sFlt-1 in preeclamptic endothelial dysfunction [7,8]. For that reason, in this study, we aimed to investigate VEGF and sFlt-1 expression in preeclamptic and normotensive placenta in a comparative manner.

\section{Materials and Methods}

\subsection{Study Design}

The ethical permission for the study was received from the ethics committee of Dicle University Faculty of Medicine. A total of twenty term placentas, of which 10 were preeclamptic and 10 were normotensive, were obtained from the obstetrics clinic of Dicle University Hospital. The demographics of women whose placentas were used in this study are shown in Table-1. Term placentas were fixed in $10 \%$ formalin and brought to the laboratory. Total placentas were dissected into small pieces and kept in fixation for 36 hours. Routine histological tissue processing protocol was performed and samples were embedded into paraffin. Serial sections of 5- $\mu \mathrm{m}$ thickness were obtained with a rotary microtome. Some of the sections from each placenta were stained with H\&E for histopathological examination. Also, VEGF and sFlt-1 immunohistochemistry was performed on the sections. 
Table 1. Demographics of normotensive and preeclampsia mothers are included in this study. Values were expressed as mean \pm SD.

\begin{tabular}{lcc}
\hline & $\begin{array}{c}\text { Normotensive } \\
(\mathrm{n}=10)\end{array}$ & $\begin{array}{c}\text { Preeclampsia } \\
(\mathrm{n}=10)\end{array}$ \\
\hline Age & $28.9 \pm 3.5$ & $31.4 \pm 5.1$ \\
Gravida & $4.8 \pm 2.4$ & $4 \pm 2.9$ \\
Parity & $3.2 \pm 1.2$ & $1.8 \pm 3.4$ \\
Mean systolic pressure $(\mathrm{mmHg})$ & $107.1 \pm 8.2$ & $115 \pm 4.2$ \\
Mean diastolic pressure $(\mathrm{mmHg})$ & $70 \pm 4.1$ & $76 \pm 6.8$ \\
Birth weight $(\mathrm{g})$ & $2710 \pm 420$ & $2668 \pm 210.8$ \\
Hemoglobin $(\mathrm{g} / \mathrm{dl})$ & $10.4 \pm 1.2$ & $12 \pm 1.4$ \\
Hematocrit $(\%)$ & $32.7 \pm 4.2$ & $34.1 \pm 2.1$ \\
Platelet $\mathrm{x} 103 / \mu \mathrm{L}$ & $270.2 \pm 72.3$ & $250.3 \pm 69.1$ \\
\hline
\end{tabular}

Ethical Statement: All procedures performed in studies involving human participants were in accordance with the ethical standards of the institutional and/or national research committee and with the 1964 Helsinki declaration and its later amendments or comparable ethical standards. This study is approved by the Dicle University Faculty of Medicine Ethics Committee. Approval number and date: 166; 08.9.2017.

\subsection{Hematoxylin and Eosin Staining}

Sections were deparaffinized in two series of xylene, and rehydrated in a decreasing series of alcohol, and brought to distilled water. Slides in distilled water were stained for 8 minutes at room temperature in hematoxylin. At the end of hematoxylin staining, slides were washed with tap water for 5 minutes. After washing, slides were brought to eosin and stained for $2 \mathrm{~min}$ and then slides were dehydrated in a series of increasing alcohol series, and thereafter immersed in xylene. Sections were mounted with Entellan and examined under a light microscope.

\subsection{Periodic Acid Schiff (PAS) Staining}

Identical deparaffinization and rehydration steps were performed on the slides for PAS staining. Then sections were brought to distilled water. Ready to use PAS staining kit (Bio Optica, \# 04130802A) was used and all steps were performed according to the manufacturer's instructions. PAS stained sections were mounted with Entellan and examined under a light microscope. The basement membrane of examined villous capillaries was measured via light microscope-adapted software and all obtained data were evaluated statistically.

\subsection{Immunohistochemistry}

Deparaffinized sections were washed in PBS and antigen retrieval was performed in EDTA with microwave heating. Tissue sample sections were then brought to room temperature and washed 
with PBS. Endogenous peroxidase activity was inhibited by applying a $3 \% \mathrm{H}_{2} \mathrm{O}_{2}$ solution to the tissue sections. Immunohistochemistry was performed with a ready to use the kit, Lab Vision ${ }^{\mathrm{TM}}$ UltraVision $^{\mathrm{TM}}$ Large Volume Detection System: anti-Polyvalent, HRP (Thermo, Waltham, Massachusetts, USA). All steps of the kit components (Ultra V Block, Biotinylated Goat AntiPolyvalent, and Streptavidin Peroxidase) were performed according to the manufacturer's instructions. After $\mathrm{H}_{2} \mathrm{O}_{2}$ application and PBS wash, nonspecific bindings were blocked by Ultra V Block. VEGF (Santa Cruz, California, USA) and sFlt-1(abcam, Cambridge, UK) antibodies were diluted 1:100 in PBS. Sections were incubated with diluted antibodies overnight at $+4{ }^{\circ} \mathrm{C}$. Sections were washed with PBS after antibody incubation and Biotinylated Goat Anti-Polyvalent dropped on sections and incubated for 10 minutes at room temperature. At the end of incubation, sections were washed with PBS and incubated with streptavidin peroxidase for 10 minutes at room temperature. The incubated sections were washed in PBS, and then DAB chromogen (Thermo, Waltham, Massachusetts, USA) was applied to develop brown color at antigen-positive parts of the tissue samples. Sections were counterstained with hematoxylin and mounted with Entellan. Quantification of immunohistochemistry was performed by considering the placental villi capillary endothelial cells either positive or negative. Randomly selected 100 villi from each group were examined for quantification. The number of positive endothelial cells was converted to percentages by considering and comparing it with the total endothelial cell counts in each villus.

\subsection{Statistical Analysis}

Obtained data were evaluated by the normality test to determine whether the values were distributed normally or not. Statistical analysis was performed by the Independent Samples t-Test and $\mathrm{p}<0.05$ was considered as significant. All data were expressed as mean \pm SD (standard deviation).

\section{Results}

\subsection{Histopathological Results}

Histopathological micrographs of the normotensive and preeclamptic placentas were shown in Figure-1. In the normotensive placenta, villi were observed with regular morphology. Syncytiotrophoblast cells and villous stroma were with normal morphology. Villous capillaries were well organized with the regular basement membrane. Syncytial knots and bridges were very few but were observed to be diffuse within the normotensive placenta. On the other hand, in the preeclamptic placenta, severe morphological degenerations were observed. Hemorrhage at the intervillous region was clearly visible. Vacuolization, edema, and disorganization within the villous stroma, an increase in the number of Hofbauer cells, and perivillous fibrin deposition were detected. In the preeclampsia group, placental syncytiotrophoblasts partially had hypertrophy. Dilatation and congestion within villous capillaries and thickening of the capillary basement membrane were very widespread. 


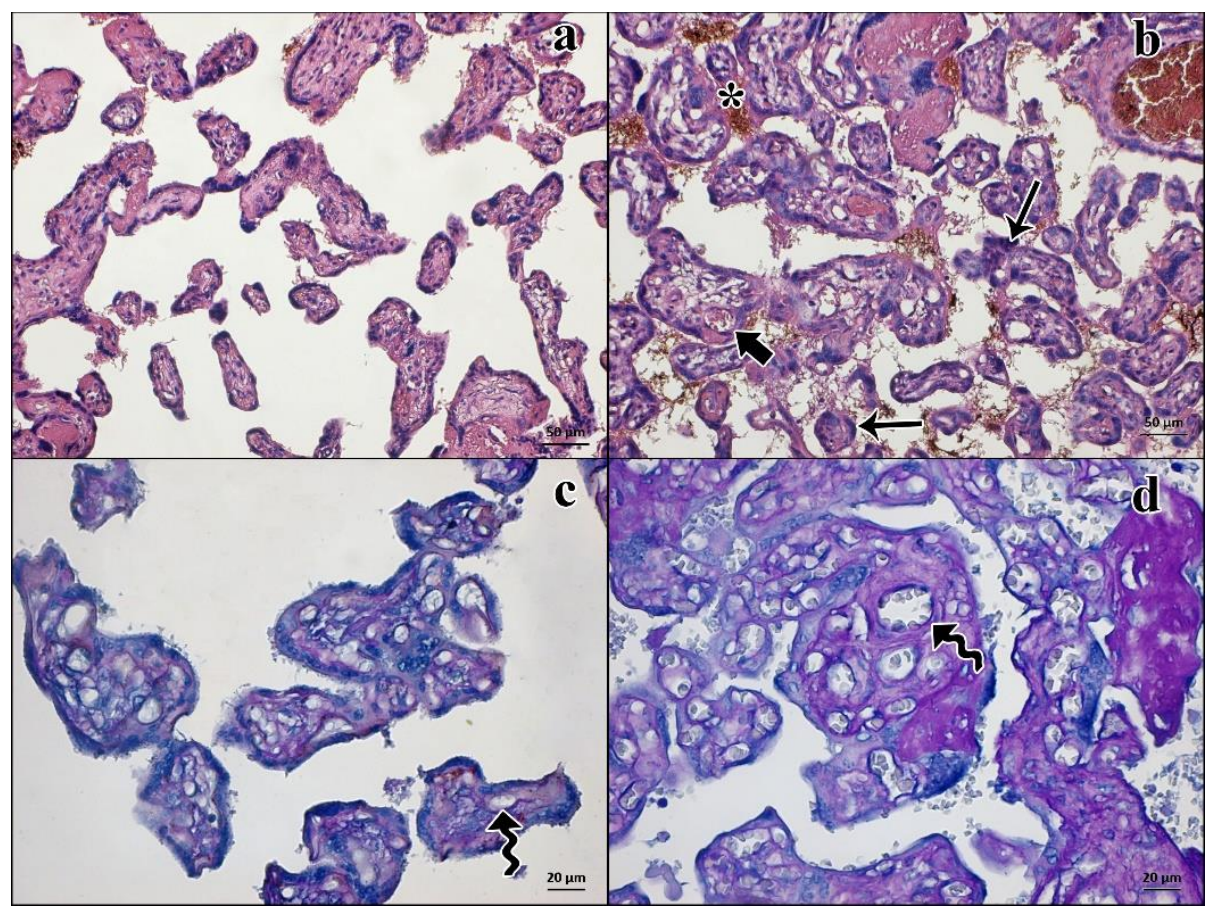

Figure 1. Histopathological micrographs of the normotensive (a,c) and preeclamptic $(b, d)$ placenta. Intervillous hemorrhage $\left(^{*}\right)$, syncytial knots and bridges (arrow), congestion in villous capillary (thick arrow), villous capillary basement membrane (curved arrow). Staining: H\&E (a,b), PAS (c,d). Bar: $50 \mu \mathrm{m}(\mathrm{a}, \mathrm{b}), 20 \mu \mathrm{m}(\mathrm{c}, \mathrm{d})$.

\subsection{Immunohistochemistry Results}

VEGF expression positivity was observed at villous capillary endothelial cells of both normotensive and preeclampsia placentas. Villous stroma was negative for VEGF, but trophoblastic cells were positive at some parts of the placenta. sFlt-1 positivity was observed in villous capillary endothelial cells, unlike negativity at stroma. Like the distribution of VEGF, syncytiotrophoblast positivity of sFlt-1 was detected in both normotensive and preeclamptic placentas. However, syncytiotrophoblast positivity was more evident in preeclamptic villi (Figure-2). 


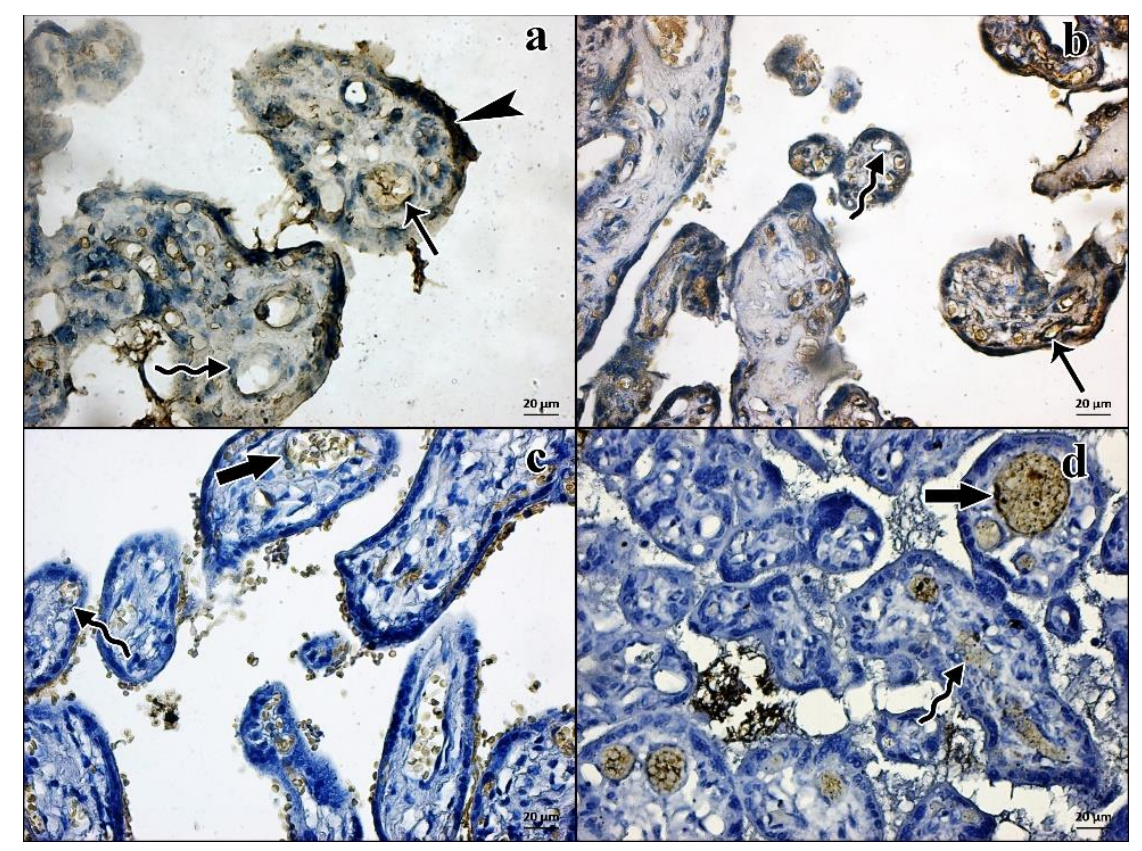

Figure 2. VEGF (a,b) and sFlt-1 (c,d) immunohistochemistry micrographs of normotensive $(a, c)$ and preeclamptic (b,d) placenta. VEGF positive (arrow) and sFlt-1 positive (thick arrow) endothelial cells. Immunonegative endothelial cells (curved arrow) and VEGF positive trophoblastic cells (arrow head). Staining: VEGF and sFlt-1 immunohistochemistry. Bar: $20 \mu \mathrm{m}$.

\subsection{Statistical Results}

Statistical results of the VEGF and sFlt-1 positivity rates were shown in Figure-3. When villous endothelial cells of normotensive placenta were evaluated, VEGF positivity rate was $50.99 \pm$ 7.17. In the preeclamptic placenta, VEGF positivity rate was $40.85 \pm 7.00$ and the difference between the groups was significant $(\mathrm{p}<0.01)$. However, sFlt-1 positivity rate of normotensive placental villous endothelial cells was $31.40 \pm 5.34$ and the positivity rate increased in preeclamptic placentas significantly $(\mathrm{p}<0.01)$ with a positivity rate of $61.96 \pm 8.20$. The mean of the villous capillary basement membrane of the normotensive placenta was $1.12 \pm 0.30 \mu \mathrm{m}$. The thicknesses of preeclamptic placental villous capillaries were $1.33 \pm 0.27 \mu \mathrm{m}$ and significantly higher $(\mathrm{p}<0.05)$ than normotensive placental villous capillary basement membrane.

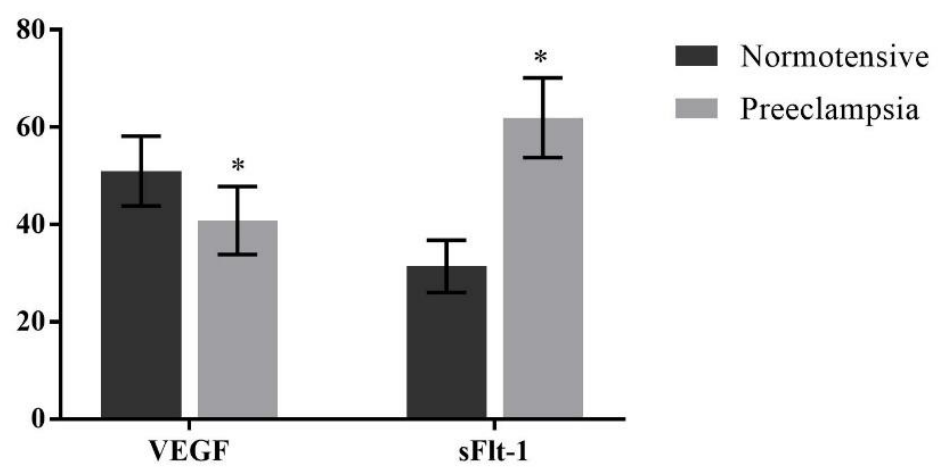

Figure 3. Statistical results of VEGF and sFlt-1 immunohistochemistry analyses. Different superscript on each bar indicates a significant difference between groups $(\mathrm{p}<0.01)$. 


\section{Discussion}

The placenta plays a role during gas and metabolite exchange between the mother and the fetus. It is also responsible for various types of metabolic activities during gestation. Failure in placental development may result in edema and proteinuria that are specific complications in preeclamptic pregnancies as well [1]. Although there are numerous studies, the underlying reasons for preeclampsia have not been understood yet. Pre-term delivery is still one of the most widely used treatment methods during preeclamptic pregnancies [9]. Recent studies suggest that VEGF and sFlt-1, which are functional in endothelial development, may be responsible for preeclampsia [10]. For that purpose, in this study, we aimed to investigate the expression of VEGF and sFlt-1 in preeclamptic placentas by immunohistochemistry.

In a previous study, Ibrahim et al. reported that there was severe morphological degeneration in preeclamptic placental villi [11]. In this study, the authors reported an increase in the number of fibrinoid depositions and syncytial knots in villi as well as reporting hemorrhage at the intervillous space. In another study, Navbir et al. reported basement membrane thickening in the preeclamptic placenta when compared to normotensive [12]. Also, villous capillary congestion was reported as a result of preeclampsia in placental histopathology [13]. In our study, we have observed severe villous degenerations like a fibrinoid deposition, an increase in the number of syncytial knots, thickening in the basement membrane of villous capillaries, and capillary congestion. In this regard, our results are consistent with previous studies. Although previous studies reported an increase in the number of villous fibrinoid depositions at the third trimester [14, 15], the tendency of the number of fibrinoid accumulations may be related to preeclampsia as reported by Ibrahim et al [11].

In a study performed by Bonnie K. Dwyer, serum sFlt-1 level was evaluated in normal and high-risk preeclamptic patients [16]. The authors highlighted that sFlt-1 level was significantly different between the groups, but the study reported a decrease in serum sFlt-1 level in high-risk patients. On the other hand, Reuvekamp et al. reported decreased serum VEGF in preeclamptic patients compared to the control group [17]. Bosio-Wheeler-Anthony et al. hypothesized that serum VEGF decrease in preeclamptic patients may be related with increase in sFlt-1[18]. In the literature review, we have found that the insoluble form of Fms Related Receptor Tyrosine Kinase 1 (Flt-1) is expressed in villous capillary endothelial cells and placental macrophages, the Hofbauer [19]. Hypertension, proteinuria, and glomerular endotheliosis were observed in pregnant rats that were subjected to adenoviral gene transfer of sFlt-1 [7]. Another study by Fan et al. reported the upregulation of sFlt-1 and VEGF in the preeclamptic placenta [20]. The results of this study indicated that sFlt-1 upregulation is related to trophoblast activation, but VEGF upregulation is related to maternal decidua. We have obtained immunohistochemistry result in the preeclamptic placenta that partially consistent with the literature. Some of the previously published studies reported increased VEGF and sFlt-1 expression in the preeclamptic placenta [21, 22]. When we consider our study, we observed reduced VEGF positivity and increased sFlt-1 positivity in preeclamptic placental villous endothelial cells. Therefore, our results are partially consistent with previous studies.

Both previous studies and our current study demonstrate that preeclampsia is related to villous endothelial dysfunction and the imbalance between VEGF and sFlt-1 might have a leading role in this process. However, we believe more and detailed studies are needed to understand the function of VEGF and sFlt-1 in the preeclamptic placenta during gestation. 


\section{Conclusion}

As a conclusion, sFlt-1 expression increased in preeclampsia patients villous capillary endothelial cells, but VEGF expression level was reduced significantly. Therefore, there is strong evidence that preeclampsia may be related to the expression level of sFlt-1 and VEGF during preeclamptic placentation.

Ethical Statement: All procedures performed in studies involving human participants were in accordance with the ethical standards of the institutional and/or national research committee and with the 1964 Helsinki declaration and its later amendments or comparable ethical standards. This study is approved by the Dicle University Faculty of Medicine Ethics Committee. Approval number and date: 166; 08.9.2017

The compliance to the Research and Publication Ethics: This study was carried out in accordance with the rules of research and publication ethics.

\section{Acknowledgment}

This work was financially supported by the Dicle University Scientific Research Projects Coordination Unit, Turkey [Tip.17.015]

\section{References}

[1] J. Roberts and D. W. Cooper, "Pathogenesis and genetics of pre-eclampsia," The Lancet, vol. 357, 9249, 53-56, 2001.

[2] U. Högberg, "The World Health Report 2005: "Make every mother and child count"-including Africans," ed: Sage Publications Sage UK: London, England, 2005.

[3] J. J. Walker, "Pre-eclampsia," The Lancet, 356(9237), 1260-1265, 2000.

[4] W. P. Mutter and S. A. Karumanchi, "Molecular mechanisms of preeclampsia," Microvascular Research, 75(1), 1-8, 2008.

[5] J. Zhang et al., "Blood pressure dynamics during pregnancy and spontaneous preterm birth," American journal of obstetrics and gynecology, 197(2), 162. e1-162. e6, 2007.

[6] E. Teran, A. Calle, and C. Escudero, "Endothelial dysfunction and preeclampsia," American Journal of hypertension, 20(9), 1026-1027, 2007.

[7] S. E. Maynard et al., "Excess placental soluble fms-like tyrosine kinase 1 (sFlt1) may contribute to endothelial dysfunction, hypertension, and proteinuria in preeclampsia," The Journal of clinical investigation, 111(5), 649-658, 2003.

[8] A. C. Palei, F. T. Spradley, J. P. Warrington, E. M. George, and J. P. Granger, "Pathophysiology of hypertension in pre- eclampsia: a lesson in integrative physiology," Acta physiologica, 208(3), 224-233, 2013.

[9] J. P. Granger, B. T. Alexander, M. T. Llinas, W. A. Bennett, and R. A. Khalil, "Pathophysiology of hypertension during preeclampsia linking placental ischemia with endothelial dysfunction," Hypertension, 38(3), 718-722, 2001. 
[10] R. Dechend and F. C. Luft, "Angiogenesis factors and preeclampsia," Nature Medicine, 14(11), 1187-1188, 2008.

[11] N. A. Ibrahim and D. M. Khaled, "Histological and immunohistochemical study on human placental tissue in normal pregnancy and preeclampsia," Cell Biol, 2, 72-80, 2014.

[12] P. Navbir, N. Alka, and G. Antima, "Histological changes in placentae in pregnancies complicated by preeclampsia and eclampsia and correlation with the fetal outcome," Int J Pharm Bio Sci, 3(2), 551-60, 2012.

[13] R. R. Corrêa et al., "Placental morphometrical and histopathology changes in the different clinical presentations of hypertensive syndromes in pregnancy," Archives of Gynecology and Obstetrics, 277(3), 201-206, 2008.

[14] I. de Luca Brunori et al., "Placental barrier breakage in preeclampsia: ultrastructural evidence," European Journal of Obstetrics \& Gynecology and Reproductive Biology, 118(2), 182-189, 2005.

[15] Z. Heidari, N. Sakhavar, H. Mahmoudzadeh-Sagheb, and T. Ezazi-Bojnourdi, "Stereological analysis of human placenta in cases of placenta previa in comparison with normally implanted controls," Journal of reproduction \& infertility, 16(2), 90, 2015.

[16] B. K. Dwyer et al., "Variable expression of soluble fms-like tyrosine kinase 1 in patients at high risk for preeclampsia," The Journal of Maternal-Fetal \& Neonatal Medicine, 23(7), 705-711, 2010.

[17] A. Reuvekamp, F. V. Velsing- Aarts, I. E. Poulina, J. J. Capello, and A. J. Duits, "Selective deficit of angiogenic growth factors characterises pregnancies complicated by pre- eclampsia," BJOG: An International Journal of Obstetrics \& Gynaecology, 106(10), 1019-1022, 1999.

[18] P. M. Bosio, T. Wheeler, F. Anthony, R. Conroy, C. O’Herlihy, and P. McKenna, "Maternal plasma vascular endothelial growth factor concentrations in normal and hypertensive pregnancies and their relationship to peripheral vascular resistance," American journal of obstetrics and gynecology, 184(2), 146-152, 2001.

[19] J. Cooper, A. Sharkey, J. McLaren, D. Charnock-Jones, and S. Smith, "Localization of vascular endothelial growth factor and its receptor, flt, in human placenta and decidua by immunohistochemistry," Reproduction, 105(2), 205-213, 1995.

[20] X. Fan et al., "Endometrial VEGF induces placental sFLT1 and leads to pregnancy complications," The Journal of clinical investigation, 124(11), 4941-4952, 2014.

[21] N. Colcimen, G. Bulut, O. Ergul Erkec, and M. Ragbetli, "Investigation of the role of vascular endothelial growth factor, Annexin A5 and Apelin by immunohistochemistry method in the placenta of preeclampsia patients," Cell Mol Biol (Noisy-le-Grand), 63, 42-45, 2017.

[22] A. Hoeller et al., "Placental expression of sFlt-1 and PIGF in early preeclampsia vs. early IUGR vs. age-matched healthy pregnancies," Hypertension in Pregnancy, 36(2), 151-160, 2017. 